\title{
HIVIAIDS: COMPLICATIONS AND CHALLENGES; THE NIGERIAN EXPERIENCE IN PEDIATRIC PRACTICE
}

\author{
D. U Nwaneri \\ Department of Child Health, University of Benin Teaching \\ Hospital (UBTH), Benin City
}

\section{INTRODUCTION}

Human Immunodeficiency Virus (HIV) is a retrovirus and belongs to the lentivirus family ${ }^{1-5}$. The HIV was first identified in 1983 and was shown to be the cause of Acquired Immune Deficiency Syndrome (AIDS) in 1984. HIV infection is characterized by the depletion of the CD4 + helper/ inducer subset of T-lymphocytes, leading to severe immune deficiency, constitutional symptoms, neurological diseases, and opportunistic infections and neoplasm (reviewed in Fauci and Lane) ${ }^{1}$. Two genetically different but related forms of HIV, called HIV-1 and HIV-2, have been isolated from patients with AIDS. HIV-1 is found worldwide and is responsible for the worldwide pandemic, and HIV-2, found mainly in West Africa, Mozambique, and Angola. HIV-2 is less pathogenic and makes little or no contribution to paediatric AIDS; therefore, all discussion in this seminar refers to HIV-1. HIV-1 has been divided into other sub types based on the genetic analysis constituent of $\mathrm{M}$ (Major) and $O$ (Outliers); hence there are subgroups or sub-types A, B, C, D, E. The following subtypes are seen in Africa: A and $D$ (East and Central), $C$ (Southern Africa in over $90 \%$ of cases), and A recombinants (West Africa). Subtype C appears to be more virulent than all the other subtypes ${ }^{6}$.
Epidemiology of HIV Infection/
Burden of the Disease

Magnitude of Paediatric HIVIAIDS in Sub-Saharan Africa

At the end of 2003, 40 million people were living with HIV worldwide. 2.1 million were children within fifteen years. Over 600,000 new cases are reported per year. Prevalence of HIV in mothers attending Antenatal clinic is $4.1 \%$ in Nigeria. In Benin 5.3\% are infected with HIV, and majority of childhood infection is via mother to child (MTCT). Children under age 15years make up $10 \%$ of the total HIV-positive population hence the need for the best available prevention, care and treatment ${ }^{7}$. The four principles of the Rights of the Child (CRC) must be taken into account; right to life, survival and development, right to be treated equally, right to participate in activities and decisions that affect them and all actions should be based on the "best interest" of the child.

Distinguishing Characteristics between Adults and Children HIVIAIDS

Transmission modes are usually different- MTCT is the predominant mode of transmission ( $>95 \%)$. This may be during pregnancy (5-10\%), labour/ delivery $(10-20 \%)$, breast feeding (5$20 \%$ ). Latency period is shorter in children. Children's immune systems are particularly prone to destruction thus leading to increase viral loads in paediatrics ${ }^{6-10}$. Body surface areabased dosing that change over time in

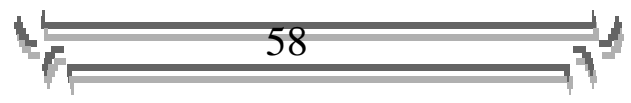


paediatrics. It is important to note that the CD4 count tends to be higher in children and it is not as reliable a tool as in adult. CD4 percentage is much more useful and in infants less than 12 months of age, neither CD4 count nor percentage is predictive of Pnuemocystic carinae pneumonia risk ${ }^{6}$, 8-9

\section{HIV Disease Progression and Outcome}

HIV disease is more aggressive than in adult. Overall MTCT rates without interventions are approximately $30-45 \%$. Untreated pediatric AIDS will die by the $2^{\text {nd }}$ birthday. The natural history of perinatally infected children fits into one of three categories:

Rapid progressors who die by age of 1 year (about 25-30\%) with onset and symptoms of AIDS within the first few months of life, median survival age 6-9 months and infections usually in-utero. It often coincide with the period of initial expansion of CD4 cells, aided by the normal movement of these cells in to the bone marrow, spleen and thymus resulting in efficient dissemination of the virus (Median value 750,000 copies/uL). Most of the patients have a positive HIV culture and/or detectable virus in plasma in the $1^{\text {st }} 48$ hours of life and the viral load rapidly increases, peaks at 2-3 months of age. Children who develop symptoms early in life and death by 3-5 yrs of age (50-60\%) usually acquired the infection perinatally. They have much slower disease progression than the rapid progressors. Survival time age of 6 years and the viral load increases by 2-3 months of age (median Value of 100,000 copies/uL). Within the next 24 months there is a decline in the viral load. Long term survivors live beyond age $8 y r s$ (5-25\%). They usually acquired the infection perinatally and they have minimal or no disease progression. They have a relatively normal CD4 cells with a very low viral load for up to or even more than 8 years ${ }^{9-11}$. The rate of downhill progression is dependent on the level of care, nutrition, and concomitant infection (example TB).

\section{Complications of HIV Infection in Children}

\section{Pulmonary Conditions in HIV}

Pneumonia and chronic lung diseases contribute to the increased morbidity and mortality of HIV-infected children. Most children present with recurrent bacterial pneumonias, but in children less than 1 year of age PCP also contributes to the high infant mortality.

Pulmonary conditions complicating HIV in children include:

\section{TB-HIV Co-infection}

The HIV pandemic has led to a resurgence of TB in both adults and children. Children also have an increased risk of developing primary progressive TB because of the associated severe immune suppression resulting from their young age and HIV. Extrapulmonary TB is seen more often in HIV-infected children. There is a higher case fatality rate for children who are co-infected with TB and HIV. The reported seroprevalence of HIV in children with TB ranges from 10 to $60 \%$ (Most common in South Africa and Nigeria). Nigeria has the $4^{\text {th }}$ largest TB and $3^{\text {rd }}$ largest HIV infection worldwide. In UBTH: $20 \%$ of those with HIV have PTB (Sadoh and Oviawe) ${ }^{6-7}$.

Diagnosing TB in children was difficult even before the HIVIAIDS pandemic; now it is more difficult because an HIVpositive child may have many other

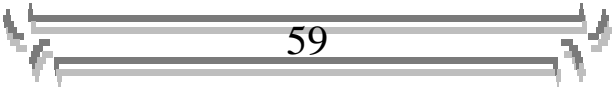


pulmonary conditions and HIV-related chronic lung diseases that mimic the symptoms of TB. Most TB diagnostic criteria (chronic symptoms, smear positive contact, positive Mantoux, response to treatment) have lower sensitivity and specificity in a co-infected child than in a non-HIV-infected child.

\section{Treating TB IN Children}

In most instances treatment of TB is usually presumptive because it is difficult to make an etiological diagnosis. In treating children who are co-infected with HIV and TB, use national guidelines

\section{Challenges in Management TB-HIV Co-infection}

Rifampicin may interact with NNRTI and PI drugs. These drugs have similar routes of metabolism and elimination (cytochrome P450 enzyme pathway). Extensive drug interactions may result in sub-therapeutic drug levels. Preferred first line is triple NRTI- ABC/ 3TC/AZT. EFV may also be used to replace NVP in standard first line. EFV not recommended in children < 3yrs of age (causes hallucinations, nightmare day dreaming). Standard first line may also be used (if possible after anti-TB therapy) ${ }^{12}$.

\section{Immune Reconstitution Syndrome in the Context of Co-therapy}

This is the paradoxical clinical deterioration after starting HAART, resulting from improved immune system interaction with organisms (TB, PCP, CMV, Herpes, cryptococcus, candida, aspergillosis) that have colonised the body during early stages of the disease. Commonly observed in patients receiving anti-TB who were initiated on ART. Usually occur within few weeks of commencement of ART. Patients usually have very low CD4 counts. Seen mainly in adults but also in children. Characterized by worsening disease after initial improvement with new onset of systemic symptoms especially: Fever, worsening pulmonary infiltrates, pulmonary effusion, Development of peripheral and mediastinal lymphadenopathy, and Expanding CNS lesions in tuberculomas ${ }^{12}$.

Generally, may be self-limiting lasts 10-40 days. If severe, requires short course glucocorticoids: Prednisone $1-2 \mathrm{mg} / \mathrm{kg} / \mathrm{d} \times 1-2$ weeks. Prevention is to start TB therapy first.

\section{Pneumocystis jiroveci pnuemonia}

Pneumocystis pneumonia (PCP) is caused by a fungus called pneumocyctis jiroveci (formerly called pneumocystis carinii). PCP is a major cause of severe pneumonia (15-30\%) and death (30$50 \%$ ) in HIV-infected infants. Infants are usually in a good nutritional state and may not have clinical features that indicate the presence of HIVIAIDS. The incidence of PCP is highest during the first year of life and usually peaks at 3 to 6 months of age though it can occur after 1 year of age, but with decreasing frequency ${ }^{6}$.

Clinical Features of PCP include Lowgrade fever or may be afebrile, marked respiratory distress (chest in-drawing, cyanosis, inability to drink). On auscultation, the chest is clear or diffuses fine crepitations. There is a poor response to standard antibiotic treatment.

Investigations include Sputum induction with nasophyaryngeal aspirates or bronchoalveolar lavage may help in diagnosing PCP and this is a big challenge in this region. There are no radiological changes specific to $\mathrm{PCP}$. In

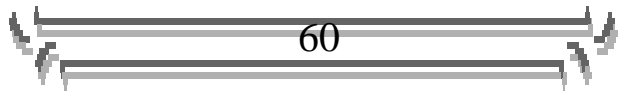


cases where a definitive diagnosis of PCP cannot be made, but where there is a high index of suspicion of PCP, therapy must be initiated promptly, along with treatment for bacterial pneumonia. Management of PCP include supportive- fluid therapy, use of paracetamol for pain; and then specific which include give intravenous high dose cotrimoxazole (CTZ) $20 \mathrm{mg} / \mathrm{kg}$ of trimethoprim per day OR $80 \mathrm{mg} / \mathrm{kg} /$ day of sulphamesoxazole given every 6 hours for 21 days. Give the same dose orally if IV preparations are not available. Add prednisone at $2 \mathrm{mg} / \mathrm{kg} /$ day for 7 to 14 day if child is in severe respiratory distress (taper if treatment $>7$ days). Other treatment modalities include Dapsone (>1 month), Pentadimine (> 5 yrs), Atovaquine ${ }^{6}$.

\section{Neurologic Complications of HIV Infections in Children}

Neurological manifestations are some of the most common modes of presentation of HIVIAIDS, but they are infrequently diagnosed in children. Delay in reaching developmental milestones, in particular, may be an early indication of HIV infection in HIV-exposed children. HIV encephalopathy is a neurologic dysfunction arising from direct HIV infection of the brain.

\section{HIV Encephalopathy}

Growth and development of the brain is outwardly manifested by achievement of childhood milestones (smiling, walking, talking, etc.). HIV infection of the brain interferes with this process and manifests as static, slowing, or regression of developmental milestones or as localized damage. This whole process is referred to as HIV encephalopathy. Age of onset of developmental delay is unpredictable, but the onset of encephalopathy may be related to the presence of other symptoms of HIV disease (e.g., hepatosplenomegaly and lymphadenopathy). HIV encephalopathy has been reported to be about $21 \%$ in HIV infected African children. $7.4 \%$ of children with HIV over a 6-year period in UBTH had HIV encephalopathy (Sadoh, Okunola and Oviawe, 2006) $)^{7}$.

Diagnosis is mainly clinical and depends on the presence of at least two of the following, for at least 2 months, in the absence of other identifiable cause, failure to attain or loss of developmental milestones or loss of intellectual ability. Decline in cognitive functions as verified by standard developmental scale or neuropsychological tests, behavioural changes, impaired brain growth or acquired microcephaly and acquired symmetrical motor deficit manifested by two or more of the following: paresis, pathologic reflexes, ataxia, or gait disturbances are other signs of HIV encephalopathy.

Challenges in Management of HIV Encephalopathy Include poor health seeking behaviours, preference to tradomedical or church care, and unaffordable cost of hospital services means patients commonly come to the hospital at advanced stage of the disease. Others include long hospital stay (mean=9.3 \pm 2.7 weeks) due to morbidity of the disease and then lack fund to pay the huge hospital bills. Advanced stage of the disease and lack of HAART contribute to high mortality rate $(80 \%$ in the study in UBTH).

\section{Malnutrition}

Childhood malnutrition is high among HIV-infected children and the magnitude is even higher in developing countries, where it is already endemic. HIV-

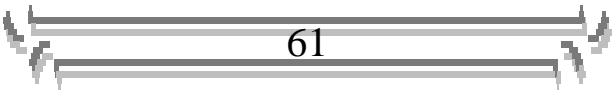


infected children are at increased risk of malnutrition for many reasons, including: decreased food intake because of anorexia associated with illness, mouth ulcers, and oral thrush. Increased nutrient loss resulting from malabsorption, diarrhoea, HIV enteropathy, increased metabolic rate because of infections, opportunistic infections, and the HIV infection itself. Release of cytokines (TNF alpha, cachetin) into plasma or tissues may mediate weight loss in HIVinfected children. The effects of malnutrition are compounded by the high burden and recurring infections and infestations in HIV-infected children. In addition, HIV-positive mothers have higher rates of low-birth-weight babies and premature birth, which are risk factors for malnutrition. Characteristics of HIV-infected children associated with malnutrition include: Micronutrient deficiencies (low serum levels of zinc, selenium, vitamins, $A, E, B 6, B 12$ and C) are common among HIV-infected children, reduce immunity, and predispose them to more infections and worsening nutritional status ${ }^{11-14}$.

\section{Challenges in Management of Paediatrics HIVIAIDS}

\section{Prevention of MTCT of HIV}

Health seeking challenges -more pregnant women need VCT. Other challenges include infant feeding problems either as prolonged breastfeeding culture and /or mixed feeding problems. Transmission rate is $3 \%$ in UBTH (unpublished data). Thanks to PEPFAR, GON etc ${ }^{7-8}$.

Diagnosis: Challenges include stigmatization, sero-discordant couples, and disruption of family unit, screening of other sibling and disclosing diagnosis to the child.

\section{Treatment of HIV in Children-Challenges}

Fewer medicines are available in paediatric formulations (Problem with dosage and compounding the drugs). The drug intake may affect future height of child in addition to weight. Change in dosage with change in weight of the child, developmental issues are critical in paediatrics, and adherence issues pose more challenges in management of paediatrics AIDS. Parent/caregiver must remember to give the drug; the child may not take the drug because of stubbornness or bad taste multiple caregivers may not communicate well concerning the drugs. There may be overdose of the child and the child may run out of drugs faster. There may be an assumption that another caregiver has already dispensed the drug.

Most importantly when to disclose to the child his/her HIV status and the consent on why he/she is taking the medicine.

Drug side effect is another challenge. Zidovudine causes anaemia, Nevirapine Hepatotoxicity, Efavirenz causes day dreaming, night mares, hallucinations in children less than 3 years old There is the problem with dosing (Doses in surface area) as well as problem with refrigeration especially stavudine.

\section{HIVIAIDS Orphans}

In Nigeria, AIDS orphans are on the increase. In 2003, 1 million AIDS orphan and the death of parents living with HIVIAIDS and the changing socioeconomic outlook may affect the number of AIDS orphan and their care. In UBTH, 13.7\% were AIDS orphans (An 8 year review of children with HIV 1996-2003) - Sadoh et al. (2005) with a mean age of $49 \pm$ 35.4 months and majority were under fives. Most had stopped schooling and childhood vaccination because of illness. Death rate was $78.6 \%$ and this shows an urgent need for the government and NGOs to attend to rising AIDS orphan problem in Nigeria.

Recommendations include; dose children appropriately and prevent HIV

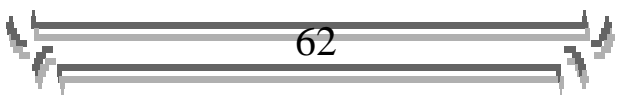


Infection in women of child bearing age. This is the best way to prevent transmission in children, strengthen antenatal care services and encourage increased attendance. Develop and promote voluntary and confidential counselling and HIV testing services. Intense implementations of PMTCT programme and make them more widespread, and then strengthened family planning services including involvement of males in family planning services.

In conclusion, Children are not adults in smaller doses! Emergence of HIV threatens to reverse the gains in child survival strategy. Challenges in Paediatrics HIVIAIDS management are enormous and therefore all hands must be on deck in eradicating this bug especially as it relates to our children.

\section{ACKNOWLEDGEMENT}

This work was supervised by $\mathrm{Dr}$ W.E. Sadoh Consultant Cardiologist, Department of Child Health, University of Benin Teaching Hospital, Benin City

\section{REFERENCES}

1. Fauci AS, Lane HC. Human Immunodeficiency Virus (HIV) disease: AIDS and related disorders. In: Fauci AS, Braunwald BE, Isselbacher $\mathrm{KJ}$, eds. Harrison's principles of internal medicine. 14th ed. New York: McGraw-Hill, 1998:1791-1856.

2. Update: mortality attributable to HIV infection among persons aged 25-44 years--United States, 1994. MMWR 45: 121, 1996.

3. Quinn TC: Global burden of the HIV pandemic. Lancet 348:99, 1996.

4. A. Balter M: Global program struggles to stem the flood of new cases. Science 280:1863, 1998.
5. Royce RA, et al: Sexual transmission of HIV. N Engl J Med 336:1072, 1997.

6. Handbook on Paediatric AIDS in Africa by the ANECCA by AIDS. 2004, pages 97-118, 121-140.

7. Sadoh WE, Okuola PO, Oviawe O. Neurologic manifestations of childhood AIDS in Benin City, Nig. Journal of pediatrics neurology 5 (2007) 21-25 IOS press.

8. Sadoh WE, Oviawe O, Jonathan A. The socio-demographic factors of children orphaned by AIDS in Benin City, Nig. Sahel medical Journal Vol 8 No. 3 July-Sept, 2005 (76-78).

9. Wizinia $A A$, et al: Pediatric HIV infection. Med Clin North Am 80:1309, 1996.

10. Geberding JL, et al: Risk of transmitting the human immunodeficiency virus to health care workers exposed to patients with AIDS and AIDSrelated conditions. J Infect Dis 156:1, 1987.

11. O'Brien J, Goedert JJ: HIV causes AIDS: Koch's postulates fulfilled. Curr Opin Immunol 8:613, 1996.

12. Paediatrics Anti-retroviral Therapy Training/ Workshop. Institute for Human Virology- Nigeria action project, Abuja, 24-28 ${ }^{\text {th }}$ July, 2006.

13. Hardy WD: The human immunodeficiency virus. Med Clin North Am 80:1239, 1996.

14. Barre-Sinoussi F: HIV as the cause of AIDS. Lancet 348:31, 1996. 